\title{
Modification of the Bonora Damage Model for shear failure
}

\author{
Gabriel Testa, Andrew Ruggiero, Gianluca Iannitti, Nicola Bonora, Domenico Gentile \\ University of Cassino and Southern Lazio, Cassino, Italy \\ gabriel.testa@unicas.it, bttp://orcid.org/0000-0001-2345-6789
}

\begin{abstract}
The Bonora damage model was extended to account for shearcontrolled damage. To this purpose, a new definition for the damage dissipation potential in which an explicit dependence on the third invariant of deviatoric stress was proposed. This expression leads to damage rate equation in which two contributions, the first for void nucleation and growth damage process the latter for shear fracture, can be recognized. For the $J_{I I I}$ controlled damage contribution, only two additional material parameters are necessary of easy experimental identification The extended model formulation was verified predicting the failure locus for AL 2024-T351 alloy. Finally, the failure locus for stress state combinations, where the minimum material ductility is expected, was determined.
\end{abstract}

KeYwords. CDM; Ductile damage; Failure locus; Triaxiality; Lode parameter; Shear fracture.

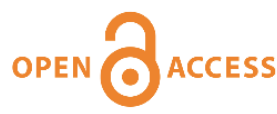

Citation: Testa, G., Ruggiero, A., Iannitti, G., Bonora, N., Gentile, D., Modification of the Bonora Damage Model for shear failure, Frattura ed Integrità Strutturale, 44 (2018) $140-150$.

Received: 11.02 .2018

Accepted: 18.03 .2018

Published: 01.04.2018

Copyright: (C) 2018 This is an open access article under the terms of the CC-BY 4.0, which permits unrestricted use, distribution, and reproduction in any medium, provided the original author and source are credited.

\section{INTRODUCTION}

7 he key role of stress triaxiality on ductile fracture of metals and alloys is well known. Rice and Tracy [1] and McClintock [2] were among the firsts to observe that the higher the stress triaxiality the smaller the strain to failure. Later, this concept was demonstrated experimentally by a number of experimental studies [3-5]. Presently, there are compelling evidences that at low or even negative triaxiality numerous classes of materials and alloys show a reduction of failure strain because of an increased susceptibility to shear fracture. McClintock [6] reported cases where ductility is terminated by shear localization and shear cracking. Johnson and Cook [5] reported a fracture strain for 4340 steel obtained from a torsion test $(\mathrm{T}=0)$ that is well below fracture strains at significantly higher mean stresses obtained under axisymmetric conditions from notched tension specimens. Later, Bao and Wierzbicki [7] showed that experimental data at failure for AL2024-T351, for different classes of stress states, falls on two distinct branches of a curve on the stress triaxiality vs ductility plot. More recently, Barsoum and Faleskog [8] also reported susceptibility to fracture under low triaxiality shearing in both mid-strength and high strength Weldox steels under combined tension-torsion loading conditions. These results indicate that stress triaxiality alone is inadequate to describe the effect of multiaxial stress state on material ductility, suggesting that also the third invariant of the stress tensor has an influence. In the last decades, several models have been proposed to account for stress triaxiality effect on material ductility. Gurson proposed a model based on the growth of a single isolated spherical void in a ductile matrix. He derived a porosity modified yield criterion in which the softening, due to void growth, is enhanced by stress triaxiality [9]. This model has been used extensively to 
predict the occurrence of ductile fracture in samples and components [10] and it still used in number or research studies. In continuum damage mechanics $(\mathrm{CDM})$, the influence of stress triaxiality is obtained from the definition of the damage energy dissipation under a generic multi-axial state of stress. In the framework of CDM, several damage model have been proposed. Lemaitre [11] developed the theoretical framework for CDM and proposed a simple linear law for damage evolution with plastic strain. Tai and Yang [12], modified the original Lemaitre model formulation introducing an exponential law for damage evolution. Later, Bonora [13] proposed an expression for the damage dissipation potential that allows obtaining a general non-linear law of evolution for damage suitable to describe ductile damage in different classes of metals. In the last decades, the Bonora damage model (BDM) was validated extensively for different materials and practical engineering cases. Iannitti et al. $[14,15]$ used the BDM to explain while ductile damage cannot occur in Taylor impact cylinder test of highly ductile metals (OFHC ad AL 1100-O) while, because of the different stress triaxiality state, it does occur in symmetric Taylor impact test (rod-on-rod) under equivalent velocity conditions. The BDM was also used to predict ductile tearing initiation and propagation in structural components such as deep water offshore pipeline welds [16-19]. Both Gurson and CDM based model formulations fail in predicting fracture under shear-controlled fracture condition. In particular, CDM models predict that material ductility has to increase when the stress triaxiality is reduced, with a maximum failure strain for pure torsion (zero stress triaxiality). In shear fracture sensitive materials, this behavior is contradicted by experimental results. For instance, in upsetting tests of AL 2024 or Ti-6Al-4V, fracture under compressive load (and negative stress triaxiality) is observed to occur at low strain, without barreling in the sample. Recently, the attempt to extend current damage model formulations to account for the influence of $\mathrm{J}_{\text {III }}$, was pursued mainly for the Gurson model. Among all, Nahshon and Hutchinson [20] proposed to add a Lode parameter dependent term in the Gurson model original formulation without the need to reformulate the model for the stress triaxiality controlled part. For what concerns CDM, only few examples of model formulation modification to incorporate shear effect can be found in the literature. Cao et al. [21] modified the Lemaitre damage dissipation potential introducing an explicit dependence on the Lode parameter. In their approach, stress triaxiality and Lode parameter act simultaneously on the damage rate making difficult to exclude a priori mutual influence between material model parameters. In this work, following the considerations that motivated the work of Nahshon and Hutchinson [20], the BDM was modified formulating a new expression for the damage dissipation potential. The novelty of the proposed extended BDM is that the damage dissipation potential is a positive definite function, which is a mandatory requirement in CDM, and that it allows to separate between stress triaxiality and shear controlled damage contributions which allow preserving all features of the original BDM formulation. The proposed extended BDM (hereafter indicated as XBDM) has been used to reproduce the variability of reported fracture strain for AL 2024 alloy over a wide range of tress triaxiality including the combined loading regime. The possibility to identify experimentally the additional material parameters is discussed.

\section{DAMAGE MODEL DEVELOPMENT}

\section{The Bonora damage model}

7 he Bonora damage model (BDM) is derived according to the thermodynamics framework of continuum damage mechanics (CDM) initially introduced by Lemaitre [22]. The CDM framework consists of three parts: the first is the definition of the state variables, which establishes the present state of corresponding physical mechanisms; the second is the definition of the state potential, from which one can derive the state laws, and the definition of associated variables; the third is the definition of the potential of dissipation to derive the evolution law of state variables, which are associated with the dissipative mechanisms. For what concerns damage processes, the state variable $D$ is introduced. Under the assumption of isotropic damage, $D$ is a scalar defined as the ratio between the damaged and the nominal net resisting area of the material reference volume element (RVE),

$$
D=\frac{A_{D}}{A_{0}}
$$

$D$ ranges from 0 , for the material in the undamaged state, to $D_{c r}$ at rupture when the material load carrying capability is completely lost. Under the strain equivalence hypothesis, the following definition for the "effective stress" is obtained,

$$
\tilde{\sigma}_{i j}=\frac{\sigma_{i j}}{(1-D)}
$$


This is the stress that should be applied to an undamaged material to cause the strain occurs in the damaged material under the nominal stress. In CDM, the effective stress replaces the Cauchy stress in the set of constitutive equations to account for damage effect on the macroscopic behavior of the material.

The use of a state variable requires the introduction of the associated state variable. The variable associated to damage is the damage strain energy density release rate $Y$, which is derived from the state potential [23],

$$
Y=\frac{\bar{\sigma}}{(1-D)} \frac{R_{v}}{2 E}
$$

Here, $\mathrm{E}$ is the Young's modulus and $\mathrm{R}_{v}$ is the function that accounts for stress triaxiality effects,

$$
\mathrm{R}_{v}=\frac{2}{3}(1+v)+3(1-2 v) \mathrm{T}^{2}
$$

where $v$ is the Poisson's ratio and $\mathrm{T}$ is the stress triaxiality factor defined as the ratio of the means stress $\sigma_{m}$ and equivalent von Mises stress $\bar{\sigma}$,

$$
\mathrm{T}=\frac{\sigma_{m}}{\bar{\sigma}}
$$

The kinetic laws of evolution are obtained from the dissipation potential. Because plastic flow can occur without damage and, similarly, damage can occur without noticeable macroscopic plastic flow, it can be assumed that the dissipation potential for plastic deformation and damage are independent,

$$
F=f+F_{D}
$$

where $f$ and $F_{D}$ are the plastic potential (also the yield function in associative flow) and the damage dissipative potential, respectively. From the generalized normality rule, the damage evolution law is obtained as follow,

$$
\dot{D}=\dot{\lambda} \frac{\partial F_{D}}{\partial Y}
$$

where $\dot{\lambda}$ is the plastic multiplier equal to the equivalent plastic strain rate scaled by damage effect,

$$
\dot{\lambda}=\dot{p}(1-D)
$$

For the damage dissipation potential, Bonora proposed the following expression,

$$
F_{D}=\left[\frac{1}{2}\left(\frac{Y}{S_{0}}\right)^{2} \frac{S_{0}}{1-D}\right]\left[\frac{\left(D_{c r}-D\right)^{\frac{\alpha-1}{\alpha}}}{(\hat{p})^{\beta}}\right]
$$

which is a convex function of the associate variables to ensure fulfillment of the second thermodynamics principle. Here, $S_{0}, \alpha, \beta$ are material constants while $\hat{p}$ is the "active plastic strain" defined as the equivalent plastic strain accumulated under positive triaxiality of the state,

$$
\hat{p}=\left\langle\Sigma_{\mathrm{T}}\right\rangle \sqrt{\frac{2}{3} \int d \varepsilon_{e q}^{p} d \varepsilon_{e q}^{p} d t}
$$


$(\ldots)$ is the Heaviside function that is equal to 1 when the stress triaxiality is positive and 0 otherwise. Under compressive state of stress, damage does not accumulate and its effects are temporarily restored $(\dot{D}=0 \& D=0)$. This provides a more consistent unilateral condition for damage accumulation and effects. Finally, from eqn. (7) and (9), assuming a power law for the material flow curve, the following expression for the damage rate is obtained,

$$
\dot{D}=\alpha\left[\frac{D_{c r}^{1 / \alpha}}{\ln \left(\varepsilon_{f} / \varepsilon_{t b}\right)}\right] R_{v}\left(D_{c r}-D\right)^{\frac{\alpha-1}{\alpha}} \frac{\dot{\hat{p}}}{\hat{p}}
$$

In this expression $\alpha, D_{c r}, \varepsilon_{t h}, \varepsilon_{f}$ are the material damage parameters where $\varepsilon_{t b}$ is the plastic strain threshold under uniaxial state of stress at which damage process is initiated, $\varepsilon_{f}$ is the failure strain under constant stress triaxiality equal to $1 / 3$, and $\alpha$ is the damage exponent that defines the shape of damage evolution law as a function of the active plastic strain.

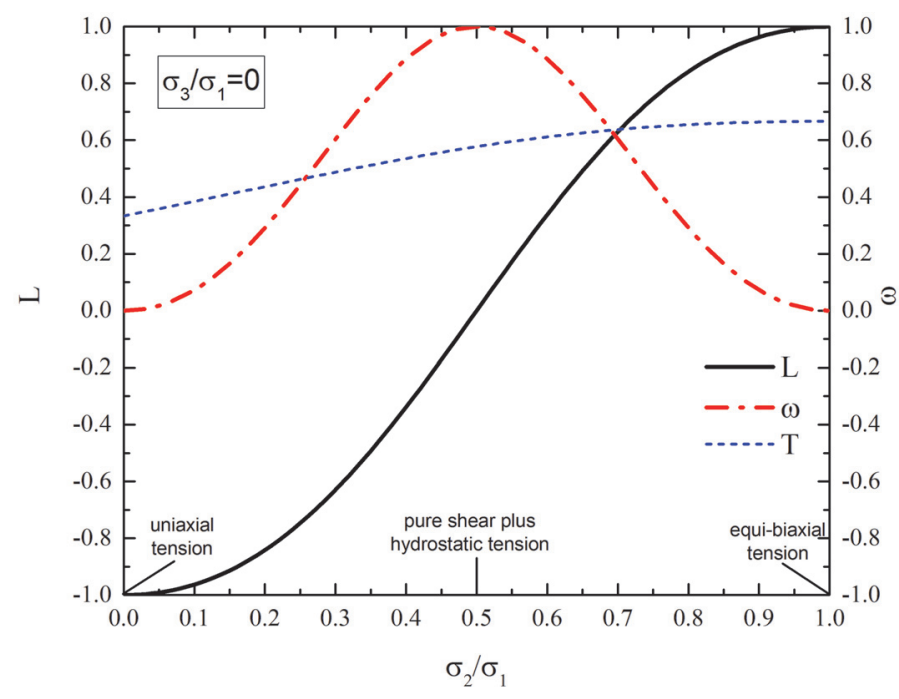

Figure 1: Evolution of L, $\omega$ and T with normalized stress ratio $\sigma_{2} / \sigma_{1}$ under plane stress condition.

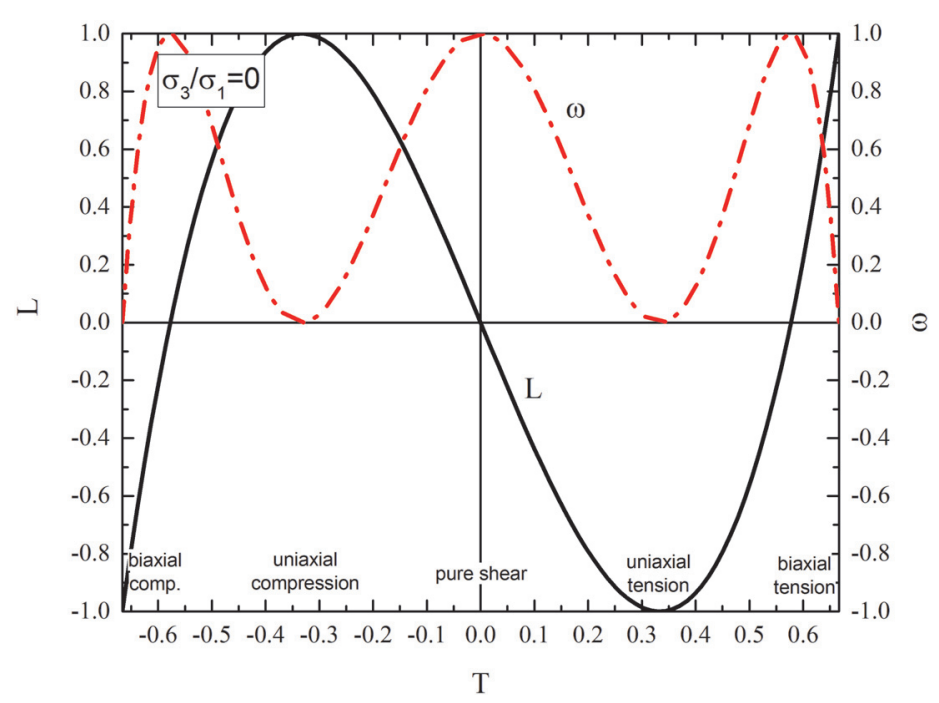

Figure 2: Evolution of $\mathrm{L}$ and $\omega$ with stress triaxiality T, under plane stress assumption (for negative T, $\sigma_{2}=0, \sigma_{3} \neq 0$ ). 


\section{Lode-dependent modified Bonora damage model for shear-dominated loading}

Although the CDM framework is not derived for a specific micromechanism, however, the underlying micromechanism is the nucleation and growth of voids (NAG). In this perspective, the failure locus predicted, by eqn. (27), is consistent with ductile failure occurring by necking of intervoid ligament. The motivation for a modification of the BDM for shear dominated loading stems from the consideration that the volume of microvoids undergoing shear may not increase but void deformation and reorientation contribute to the loss of load carrying capability and constitute an effective increase in damage. Under shear-dominated loading, the stress triaxiality is zero or slightly positive and current BDM predicts an increase of damage at the slowest rate which implies the largest ductility that material can exhibit. The Lode parameter has been introduced to account for the influence of the third invariant of stress on material ductility,

$$
L=-\frac{27}{2} \frac{J_{3}}{\bar{\sigma}^{3}}
$$

where $J_{3}$ is third invariant of the deviatoric stress tensor $s_{i j}$,

$$
J_{3}=\operatorname{det}\left(s_{i j}\right)=\frac{1}{3} s_{i j} s_{i k^{3}} s_{j k}=\left(\sigma_{1}-\sigma_{m}\right)\left(\sigma_{2}-\sigma_{m}\right)\left(\sigma_{3}-\sigma_{m}\right)
$$

The following expression for the Lode parameter, as a function of the stress ratios $a=\sigma_{2} / \sigma_{1}$ and $b=\sigma_{3} / \sigma_{1}$ can be written,

$$
L=-\frac{1}{2} \frac{(a-2 b+1)(2 a-b-1)(a+b-2)}{\left[a^{2}+a b-a+b^{2}+b+1\right]^{3 / 2}}
$$

For plane stress $\left(\sigma_{3}=0, b=0\right)$, it reduces to,

$$
L=-\frac{1}{2} \frac{2 a^{3}-3 a^{2}-3 a+2}{\left[a^{2}-a+1\right]^{3 / 2}}
$$

The Lode parameter is bounded between -1 and 1 : $\mathrm{L}=-1$ for uniaxial tension $(a=b=0)$, while for equibiaxial plane stress tension $(a=1, b=0) \mathrm{L}=1$ and for pure shear stress $(a=0, b=-1), \mathrm{L}=0$. Under plane stress condition, the expression of the Lode angle as a function of the stress triaxiality can also be uniquely determined,

$$
L=-\frac{27}{2} T\left(\frac{1}{3}-T^{2}\right)
$$

Nahshon and Hutchinson [20] introduced the following parameter,

$$
\omega=1-L^{2}
$$

The evolution of $L$ and $\omega$ with the stress ratio $a$ and $T$, for plane stress condition, is shown in Fig. 1 and Fig. 2 respectively. In the latter figure, $\mathrm{L}$ vs $\mathrm{T}$ is plotted over the stress triaxiality range of significance $[-1 / 3,1 / 3]$ under plane stress assumption. In order to extend current BDM model formulation for stress states centered on a pure shear stress plus a hydrostatic contribution, the original expression of the damage dissipation potential is modified, similarly to [20], introducing a dependency on $\omega$ and that does not vanish when $\sigma_{m}=0$ as follow,

$$
F_{D}=\left[\frac{1}{2}\left(\frac{Y}{S_{0}}\right)^{2} \frac{S_{0}}{1-D}\right]\left[\frac{\left(D_{c r}-D\right)^{\frac{\alpha-1}{\alpha}}}{(\hat{p})^{\beta}}\right]+\left[\left(\frac{Y}{S_{0}}\right) \frac{S_{0}}{1-D} A_{\omega} \omega^{k} D^{\beta}\right]
$$




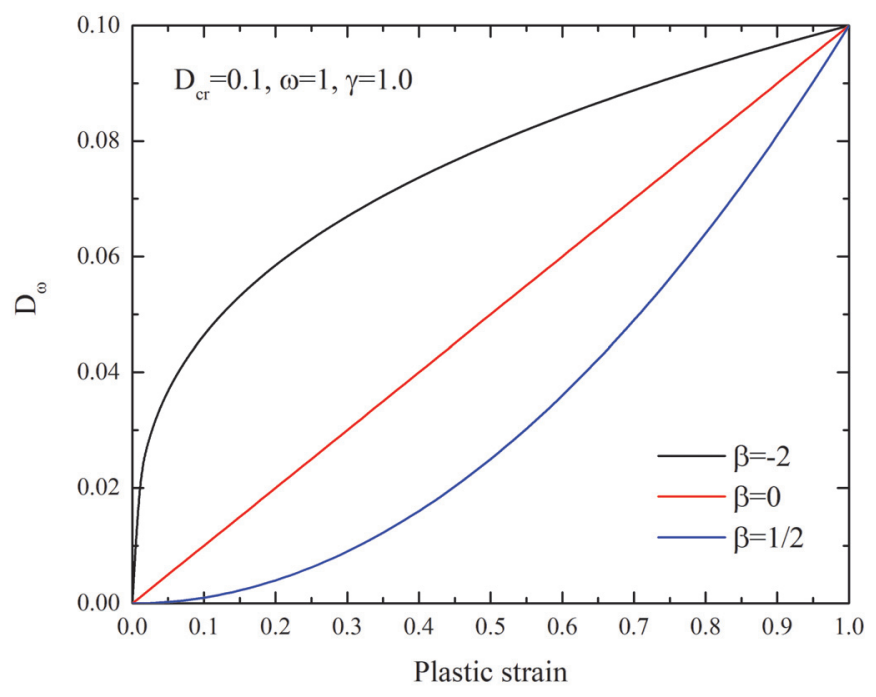

Figure 3: Damage evolution for different values of the shape factor $\beta,(k=1)$.

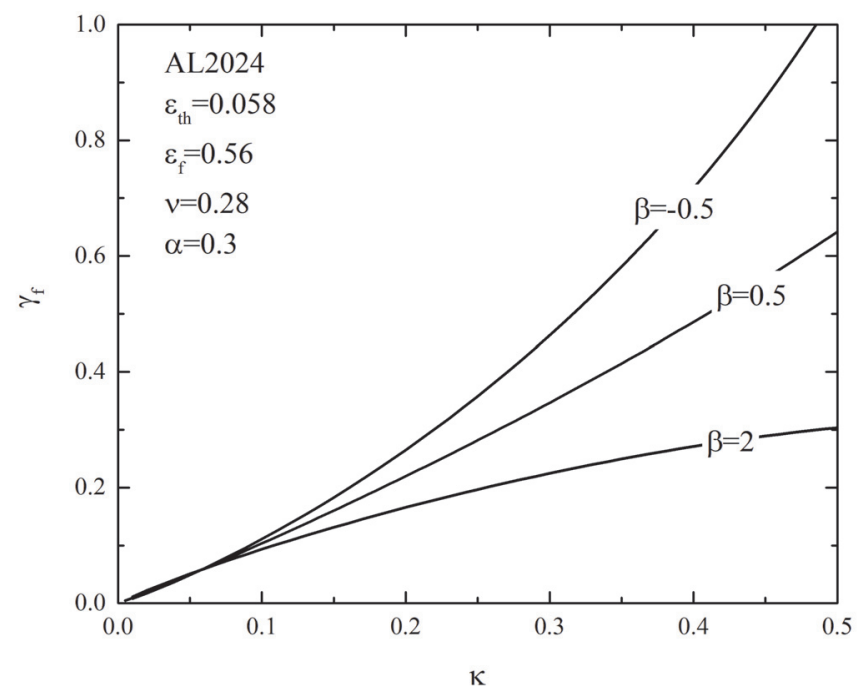

Figure 4: Relationship between the critical shear strain $\gamma_{f}$ and the torsional fracture strain $\kappa$.

This expression satisfies the requirement of being a positive definite function. Here, no coupling between $\mathrm{T}$ and $\omega$ dependent terms is assumed. Thus, the damage rate can be separated in two contributions: the first for NAG governed by stress triaxiality and the latter, for shear controlled fracture, governed by $\omega$,

$$
\dot{D}=\dot{\lambda} \frac{\partial F_{D}}{\partial Y}=\dot{D}_{\mathrm{T}}+\dot{D}_{\omega}
$$

where $\dot{D}_{\mathrm{T}}$ is given by eqn. (11). Recalling eqn. (7) and eqn. (8), the following expression for $\dot{D}_{\omega}$ is obtained,

$$
\dot{D}_{\omega}=A \omega^{k} D_{\omega}^{\beta} \dot{p}
$$

The simple power law dependence on the parameter $\omega$ is justified by Barsoum and Faleskog [8] findings. These indicate that the presence of voids do not seem to play a major role for predicting ductile failure at low levels of triaxiality while 
failure seems to be governed by a simple criterion based on a critical measure of plastic shear deformation. Consequently, for simplicity purpose, it is assumed that shear deformation induced damage starts to accumulate as soon as plastic deformation start to occur (no threshold). Integrating eqn. (20) for $\omega=1$, the material constant $A$ can be determined as

$$
\int_{0}^{D_{\sigma r}} \frac{d D_{\omega}}{D_{\omega}^{\beta}}=A \int_{0}^{\gamma_{f}} d p
$$

from which it follows

$$
A=\frac{D_{\omega}^{1-\beta}}{1-\beta} \frac{1}{\gamma_{f}}
$$

For constant $\omega$ load paths and $\beta \neq 1$, eqn. (20) can be integrated to get

$$
D_{\omega}=D_{c r}\left[\frac{\omega^{k}}{\gamma_{f}} p\right]^{\frac{1}{1-\beta}}
$$

where $\gamma_{f}$ is the critical strain for pure shear and $\beta$ is a shape factor. Varying $\beta$, different shape of $D_{\omega}$ evolution with plastic strain can be obtained, included linear damage law for $\beta=0$. In Fig. 3, damage evolution for different values of the shape factor $\beta$ are shown. For $\beta=1$ an exponential damage law, similarly to Rice and Tracy void growth law, is obtained

$$
\frac{D}{D_{c r}}=\exp \left(-\ln \left(\frac{D_{c r}}{D_{0}}\right) \frac{\omega^{k}}{\gamma_{f}}\left(p_{f}-p\right)\right)
$$

In this case, the assumption of an initial damage $D_{0}$, in association with preexisting voids or nucleating particles, becomes necessary. For constant $\omega$ (or $L$ ) and $T$ deformation process, the damage rate equation can be integrated leading to the following expression,

$$
D=D_{c r}\left\{\left[1-\left(1-R_{v} \frac{\ln p / \varepsilon_{t h}}{\ln \varepsilon_{f} / \varepsilon_{t h}}\right)^{\alpha}\right]+\left[\frac{\omega^{k}}{\gamma_{f}} p\right]^{\frac{1}{1-\beta}}\right\}
$$

Present model formulation predicts that for torsion $(T=0$ and $\omega=1)$ there is also a damage contribution due to stress triaxiality for those materials in which the failure strain in torsion is larger than the damage threshold strain $\mathcal{E}_{t b}$. In these cases, provided the torsional failure strain (from experiment) $\kappa$, the parameter $\gamma_{f}$ can be determined for $\beta \neq 1$ as

$$
\gamma_{f}=\left[\kappa\left(1-\frac{2}{3}(1+v) \frac{\ln \kappa / \varepsilon_{t b}}{\ln \varepsilon_{f} / \varepsilon_{t h}}\right)^{-\alpha(1-\beta)}\right]
$$

In Fig. 4 the relationship between $\gamma_{f}$ and $\kappa$ for AL 2024 is shown. It is interesting to note that eqn. (26) states that the relationship between the parameter $\gamma_{f}$ and $\kappa$ depends, among all, on the shape of damage exponents $\alpha$ and $\beta$ suggesting that a possible mutual dependence between these two parameters may exists. 


\section{FRACTURE LOCUS PREDICTION}

he concept of fracture, or failure, locus (FL), or limit strain diagram (LSD), was introduced by Mackenzie et al. [24]. They analyzed the effect of the stress state on failure strain of several steels (HT80, HY130, Marrel, low carbon steel and aluminum alloys) performing traction tests on round notched bar samples. For some materials, also fracture strain in torsion is reported. In their work, stress triaxiality was estimated by means of the Bridgman solution derived for a necked bar. As confirmed several years later with the development of finite element technology, this definition of stress triaxiality may differs considerably from the effective stress triaxiality occurring in the sample especially at the failure location. However, they were among the firsts to recognize that the effect of the state of stress on fracture initiation may vary for different classes of alloys. Later, the fracture locus was also proposed as possible fracture criterion. To this purpose several phenomenological relationships, between failure strain and stress triaxiality, obtained fitting available experimental data at failure, have been proposed [25, 5]. In CDM, the fracture locus can be derived from the model formulation. In fact, equating eqn. (11) integrated for a generic proportional loading condition and for $\mathrm{T}$ equal to $1 / 3$, the material failure strain $p_{f}$ as a function of stress triaxiality can be derived

$$
\frac{p_{f}}{p_{t h}}=\left(\frac{\varepsilon_{f}}{\varepsilon_{t h}}\right)^{\frac{1}{R_{v}}}
$$

For low stress triaxiality $(\mathrm{T}<3)$, which is that resulting from geometry variations such as notches, the stress triaxiality effect on the damage threshold strain can be neglected $\left(p_{t h}=\varepsilon_{t h}\right)$, which lead to

$$
p_{f}=\varepsilon_{t b}\left(\frac{\varepsilon_{f}}{\varepsilon_{t h}}\right)^{\frac{1}{R_{v}}}
$$

According to this expression, material fracture strain increases with decreasing the stress triaxiality with a maximum for pure shear $\mathrm{T}=0$ (torsion). In the present framework, in which stress triaxiality alone is insufficient to describe the effect of the stress state on material fracture strain, eqn. (28) is valid under for $\omega=0$.

Similarly, the failure locus for shear controlled fracture can be obtained. In fact, for $T=0$, equating eqn. (20) integrated for constant $\omega$ and for $\omega=1$, we get

$$
p_{f}=\frac{\gamma_{f}}{\omega^{k}}
$$

According to this expression, the material failure strain varies hyperbolically with $\omega$. Under plane stress condition $\left(\sigma_{3}=0\right)$ the relationship in eqn. (16), between $L$ and $\mathrm{T}$, can be substituted in (29) to obtain the failure strain in the shear dominated region of the fracture locus diagram. For uniaxial and equibiaxial tension the failure strain for shear controlled fracture becomes infinite $(\omega=0)$ indicating that shear fracture cannot occurs under these stress states. For pure shear loading (i.e. torsion, $\omega=1$ ), the failure strain reaches its minimum. Under negative stress triaxiality, with a lower bound at $\mathrm{T}=-1 / 3$, the solution also predicts that failure under controlled shear can still occur while damage due to void growth cannot develop because of the unilateral condition.

\section{MODEL VALIDATION: APPLICATION TO AL2024-T351}

7 he extended Bonora damage model (XBDM) was verified predicting fracture in AL2024-T351 alloy under different stress states (different combination of $T$ and $\omega$ ). Bao and Wierzbicki [7] reported failure strain data measured on different specimen geometries: round notched (RNB) bar samples, cylinders with different diameter over height ratios under uniaxial compression, "butterfly" flat specimen for pure shear and combined loading. Although 
these tests cover a wide stress triaxiality range from $-1 / 3$ up to 1 , the Lode parameter, and consequently $\omega$, is not constant. In order to verify the predicting capability of the proposed model formulation, material model parameters for AL2024-T351 have been determined based on available experimental data. In particular, the parameters for the stress triaxiality controlled damage contribution $D_{T}$ have been determined by fitting of the experimental data relative to tractions performed on RNB specimens. For these geometries the state of stress state is axisymmetric and $\omega=0$. Thus, $\varepsilon_{t b}$ and $\varepsilon_{f}$ can be determined by fitting using the expression for the failure locus given in eqn. (28).

Similarly, the parameters for the shear controlled damage $D_{\omega}$ have been determined by fitting of the experimental data with $\mathrm{T}<0$, for which the contribution of $D_{T}$ is zero because of the unilateral condition. The damage model parameters for AL2024-T351 are summarized in Tab. 1.

\begin{tabular}{cc}
\hline$\varepsilon_{t h}$ & 0.054 \\
$E_{t h}$ & 0.53 \\
$D_{c r}$ & 0.1 \\
$\alpha$ & 0.3 \\
$\beta$ & 0.35 \\
$\gamma_{f}$ & 0.23 \\
$\kappa$ & 0.30 \\
\hline
\end{tabular}

Table 1: Summary of damage model parameters for AL 2024-T351.

In Fig. 5 the comparison of predicted fracture loci for stress triaxiality and shear dominated damage with experimental data is shown. Here, it can be noted that the agreement is very good for both regions controlled either by $\omega$ or by $T$. The gray shaded area is the region where both $T$ and $\omega$ contribute to determine the critical condition for fracture. Also pure torsion falls in this region, since according to the present model formulation, there is a contribution to damage due to $D_{T}$ for $\mathrm{T}=0$. Consequently, as expected, fracture strain predicted by eqn. (29) is larger than that measured in pure torsion test. In the intermediate region, where both $\mathrm{T}$ and $\omega$ play a role, fracture strain can be predicted under the assumption of proportional loading ( $\mathrm{T}=$ const.) and constant $\omega$ load path using eqn. (25). At fracture $D=D_{c r}$ and $p=p_{f}$, thus

$$
\left(1-R_{v} \frac{\ln p_{f} / \varepsilon_{t b}}{\ln \varepsilon_{f} / \varepsilon_{t b}}\right)^{\alpha}=\left[\frac{\omega^{k}}{\gamma_{f}} p_{f}\right]^{\frac{1}{1-\beta}}
$$

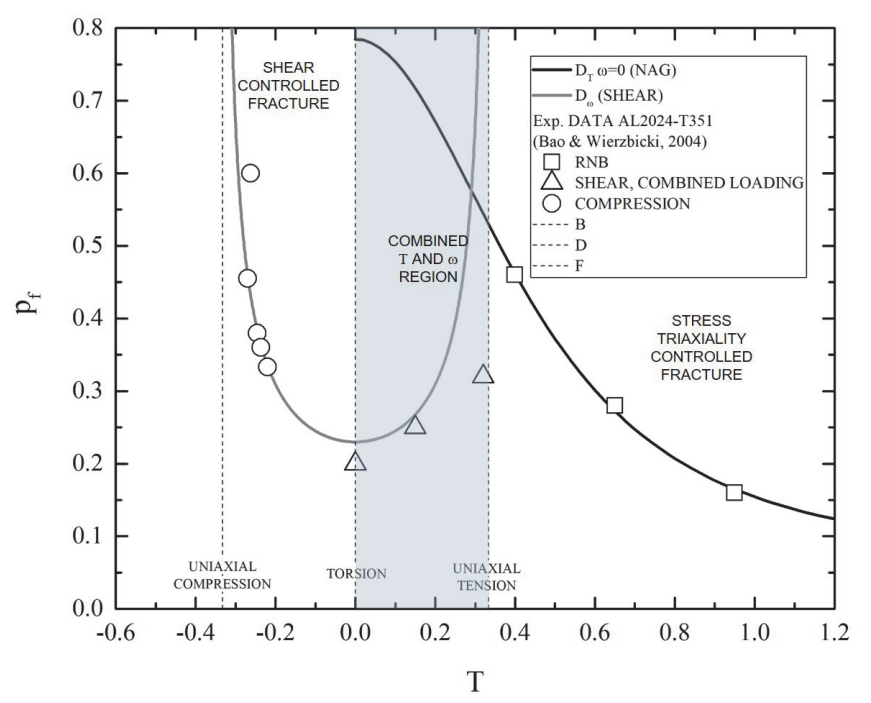

Figure 5: Comparison of the predicted failure locus with experimental data for AL2024-T351. 
This equation can be solved graphically for given $T$ and $\omega$ values to obtain $p_{f}$. In Fig. 6 , the calculated failure strain for experiments in the combined regions is plotted (square black dots). Data points have been determined using eqn. (30) provided the corresponding $\omega$ that was estimated from finite element simulation. The agreement is very good confirming the capability of the proposed modelling to predict accurately the occurrence of fracture under complex stress states.

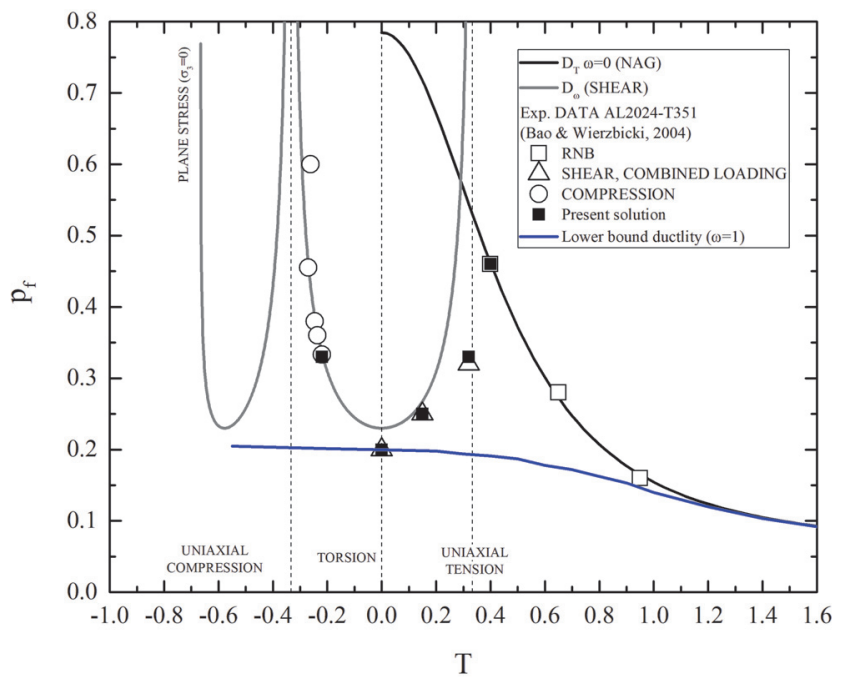

Figure 6: Failure locus or AL2024-T351: lower bound solution.

Eqn. (30) can be used to predict the regime of stress triaxiality where the material would exhibit the minimum ductility because of the maximum contribution of shear damage. In Fig. 6 the lower-bound ductility line is plotted solving eqn. (30) for $\omega=1$. It is interesting to note that this solution predicts that the reduction of ductility for stress triaxiality up to 1 approximately. For higher stress triaxiality values, the solution merges on that obtained for stress triaxiality controlled damage. This implies that increasing the stress triaxiality overrules the Lode parameter effect. This lower bound line corresponds to in plane shear combined with pressure. Under plane strain condition, such stress state can be obtained simply applying a tensile (or a compressive) stress to pure shear deformation. In this view, the plane strain ductility is then the minimum ductility that the material would exhibit for unflawed geometry, consistently with fracture mechanics.

\section{CONCLUSIONS}

$\mathrm{I}$ $\mathrm{n}$ this work, the Bonora damage model was extended to account for shear-controlled fracture. To this purpose, based on the motivations given in [20], the original expression of the damage dissipation potential was modified introducing a dependency on the third invariant of the deviatory stress. This leads to a new definition of the damage rate equation with two terms, the first controlled by stress triaxiality and the latter controlled by $\omega$ which is related to the Lode parameter. The proposed model formulation allows predicting the failure locus for selected state of stress states and to identify the region, in ductility vs stress triaxiality plot, where $J_{I I I}$ is expected to have an effect in the reduction of material ductility. Model prediction capabilities have been verified predicting fracture strain in AL 2024-T351 under different stress states. In particular, it was found that, according to the model solution, shear damage may play a role also for high stress triaxiality range $(1>T>1 / 3)$. Finally fracture is predicted to occur under negative stress triaxiality also below the "cut off" of $\mathrm{T}=-1 / 3$, provided that a compressive stress is superimposed to a pure shear deformation state.

\section{REFERENCES}

[1] Rice, J. R. and Tracey, D. M., (1969). On the ductile enlargement of voids in triaxial stress fields. Journal of the Mechanics and Physics of Solids, 17, pp. 201-217.

[2] Mcclintock, F. A., (1968). A criterion for ductile fracture by the growth of holes. Journal of applied mechanics, 35, pp. 363-371. 
[3] Hancock, J. and Mackenzie, A., (1976). On the mechanisms of ductile failure in high-strength steels subjected to multi-axial stress-states. Journal of the Mechanics and Physics of Solids, 24, pp. 147-160.

[4] Le Roy, G., Embury, J., Edwards, G. and Ashby, M., (1981). A model of ductile fracture based on the nucleation and growth of voids. Acta Metallurgica, 29, pp. 1509-1522.

[5] Johnson, G. R. and Cook, W. H., (1985). Fracture characteristics of three metals subjected to various strains, strain rates, temperatures and pressures. Engineering fracture mechanics, 21, pp. 31-48.

[6] Mcclintock, F. A. 1971. Plasticity Aspects of Fracture. Fracture: An Advanced Treatise. New York: Academic Press.

[7] Bao, Y. and Wierzbicki, T., (2004). On fracture locus in the equivalent strain and stress triaxiality space. International Journal of Mechanical Sciences, 46, pp. 81-98.

[8] Barsoum, I. and Faleskog, J., (2007). Rupture mechanisms in combined tension and shear-Experiments. International Journal of Solids and Structures, 44, pp. 1768-1786.

[9] Gurson, A. L., (1977). Continuum theory of ductile rupture by void nucleation and growth: Part I-Yield criteria and flow rules for porous ductile media. Journal of engineering materials and technology, 99, pp. 2-15.

[10] Needleman, A. and Tvergaard, V. An analysis of ductile rupture in notched bars. Journal of the Mechanics and Physics of Solids, (1984) 32, pp. 461.

[11] Lemaitre, J., (1985). A continuous damage mechanics model for ductile fracture. Journal of Engineering Material and Technology, 107, pp. 83-89.

[12] Tai, W. H. and Yang, B. X., (1987). A new damage mechanics criterion for ductile fracture. Engineering Fracture Mechanics, 27, pp. 371-378.

[13] Bonora, N., (1997). A nonlinear CDM model for ductile failure. Engineering Fracture Mechanics, 58, pp. 11-28.

[14] Iannitti, G., Bonora, N., Ruggiero, A. and Testa, G., (2014). Ductile damage in Taylor-anvil and rod-on-rod impact experiment. Journal of Physics: Conference Series, 500.

[15] Iannitti, G., Bonora, N., Bourne, N., Ruggiero, A. and Testa, (2017). G. Damage development in rod-on-rod impact test on 1100 pure aluminum. AIP Conference Proceedings,

[16] Carlucci, A., Bonora, N., Ruggiero, A., Iannitti, G. and Testa, G. (2014). Crack initiation and propagation of clad pipe girth weld flaws. American Society of Mechanical Engineers, Pressure Vessels and Piping Division (Publication) PVP,

[17] Testa, G., Bonora, N., Gentile, D., Ruggiero, A., Iannitti, G., Carlucci, A. and Madi, Y., (2017). Strain capacity assessment of API X65 steel using damage mechanics. Frattura ed Integrita Strutturale, 11, pp. 315-327.

[18] Bonora, N., Gentile, D., Ruggiero, A., Testa, G., Folgarait, P. and Calatroni, A. (2013). Failure assessment of pipe tee element using continuum damage mechanics. American Society of Mechanical Engineers, Pressure Vessels and Piping Division (Publication) PVP.

[19] Ruggiero, A., Iannitti, G., Testa, G., Limido, J., Lacome, J. L., Olovsson, L., Ferraro, M. and Bonora, N., (2014). High strain rate fracture behaviour of fused silica. Journal of Physics: Conference Series, 500.

[20] Nahshon, K. and Hutchinson, J., (2008). Modification of the Gurson model for shear failure. European Journal of Mechanics-A/Solids, 27, pp. 1-17.

[21] Cao, T.-S., Gachet, J.-M., Montmitonnet, P. and Bouchard, P.-O., (2014). A Lode-dependent enhanced Lemaitre model for ductile fracture prediction at low stress triaxiality. Engineering Fracture Mechanics, 124, pp. 80-96.

[22] Lemaitre, J., (1990). Micro-mechanics of crack initiation. International journal of fracture, 42, pp. 87-99.

[23] Lemaitre, J. (2012). A course on damage mechanics, Springer Science and Business Media.

[24] Mackenzie, A., Hancock, J. and Brown, D., (1977). On the influence of state of stress on ductile failure initiation in high strength steels. Engineering fracture mechanics, 9, pp. 167-188.

[25] Manjoine, M., (1982). Creep-Rupture Behavior of Weldments. Welding J., 61, pp. 50. 